\title{
3D Surface-based Detection of Pleural Thickenings
}

\author{
P. Faltin, K. Chaisaowong
}

\begin{abstract}
Pleuramesothelioma is a malignant tumor of the pleura. It evolves from pleural thickenings which are a typical long-term effect of asbestos exposure. A diagnosis is performed by examining CT scans acquired from the patient's lung. The analysis of the image data is a very time-consuming task and is subject to strong inter- and intra-reader variances. To objectivize the analysis and to speed-up the diagnosis a full automatic system is developed. An essential step in this process is to identify potential thickenings. In this paper we describe the complete system in brief, and then take a closer look on thickening detection. A CT slice based approach is presented here. It is extended by using 3D knowledge of the lung surface which could scarcely have been acquired visually.
\end{abstract}

Keywords: pleuramesothelioma, pleural thickenings, computer-assisted diagnosis, convex hull, morphological erosion, CT.

\section{Introduction}

Asbestos was widely used until the 1990s because of its useful properties. Today it is known to be a carcinogenous substance for humans. Fibres $3-5 \mu \mathrm{m}$ in length can get into the pulmonary alveolus and cannot be decomposed by the body. The fibers are typically accumulated on the lung boundary. The long persistence and the consequent continuous penetration of the pleura can result in pleural thickenings and hence pleuramesothelioma. Long latency of 14-72 years leads to an expected peak in diagnosed cases in 2018 [1]. High-risk patients undergo regular medical checkups, which also include the acquisition of 3D CT image data. The CT scan typically contains approximately 700 image-slices, which have to be inspected by a medical expert. This task requires much time and concentration, and is subject to strong inter- and intra-reader variances [2]. In order to support the medical experts and objectivize the diagnosis we have developed a fully automatic system. It automatically detects, analyses and measures thickenings using CT data from one point of time. The system also comprises a follow-up assessment, in which thickenings recorded at two different points in time are compared regarding their thickness and volume.

The whole process consists of several steps. In the first step, two-step supervised range-constrained Otsu thresholding [3] is used to segment the lung tissue. The next step is thickening detection and extraction, which is presented in this paper. For exact measurements of volume and thickness, the thickenings are classified as pleural plaques or as diffuse pleural thickenings. A thin plate spline interpolation [4] is applied to reconstruct the correct thickening ex- pansion, depending on the classification. To perform the follow-up assessment a rigid registration [5] between the image data of two different points in time is performed. Applying this transformation, the thickenings are matched with regard to their position and the CT number distribution in Hounsfield units [6]. In the final step, the growth rate of the matched thickenings is calculated.

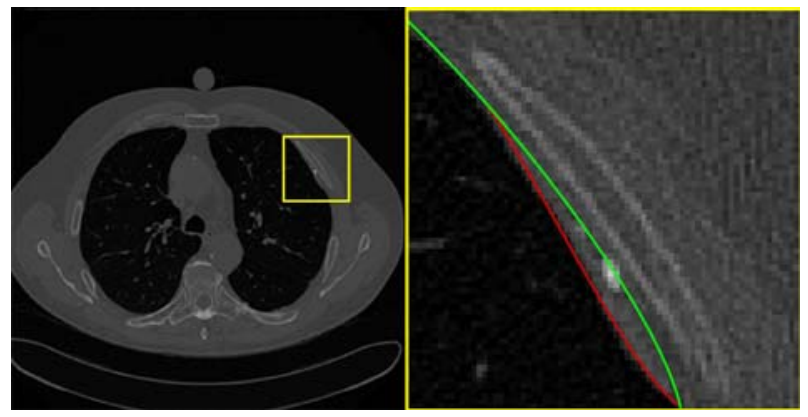

Fig. 1: An example of typical pleural thickening

\section{Methods}

The presented method is oriented towards the human perception. As shown in Figure 1, thickenings can mainly be recognized by an indention of the lung surface, visualized in red, compared to the green healthy lung boundary. The fundamental step in the thickening detection presented here is to individually model a virtual healthy lung mask $\hat{S}$ on the lattice $\boldsymbol{R}=\{(x, y, z): 1 \leq x \leq X 1 \leq y \leq Y 1 \leq z \leq Z\}$, where $X, Y, Z$ are the dimensions of the CT image. If a voxel $\boldsymbol{i} \in \boldsymbol{R}$ is located inside the lung, $S(\boldsymbol{i})=1$ applies, and if it is located outside the lung, $S(\boldsymbol{i})=0$ applies. An older approach [6] generates the healthy lung model analyzing the CT data in the transverse 
planes (here: fixed $z$ ) slice by slice. The surrounding slices are only considered for the postprocessing. Thickenings which are mainly visible from a sagittal plane (here: fixed $x$ ) or from a coronal plane (here: fixed $y$ ), as shown in Figure 2, are not reliably detected.

To overcome this uncertainty, the new method models a 3D healthy lung to detect potential thickenings. The difference between thickenings and a healthy lung surface can mainly be described by an indention of the detected lung surface in the direction of the lung center. Mathematically expressed, let $\left\{\boldsymbol{p}_{1}, \boldsymbol{p}_{2}\right\} \subset \boldsymbol{R}_{\boldsymbol{L}}$ be any two points of the thickening boundary, with $\boldsymbol{R}_{\boldsymbol{L}}=\{\boldsymbol{i}: \boldsymbol{i} \in \boldsymbol{R} ; S(\boldsymbol{i})=1\}$. The direct connection $\boldsymbol{D}=\left\{t \boldsymbol{p}_{1}+(1-t) \boldsymbol{p}_{2}: t \in[0,1]\right\}$ is not fully covered by the lung mask, hence $\exists \boldsymbol{d} S(\boldsymbol{d})=$ $0 ; \boldsymbol{d} \in \boldsymbol{D}$. This definition is similar to the definition of a convex hull, whereas $\forall \boldsymbol{d} S(\boldsymbol{d})=1 ; \boldsymbol{d} \in \boldsymbol{D}$ holds for all points $\left\{\boldsymbol{p}_{1}, \boldsymbol{p}_{2}\right\}$ of the lung mask. In a closed form, a convex hull of the lung model is given by

$$
\operatorname{conv}\left(\boldsymbol{R}_{\boldsymbol{L}}\right)=\left\{\sum_{i \in R} a_{i} \cdot \boldsymbol{i}: \sum_{i \in R} a_{i}=1 ; a_{i} \geq 0\right\} .
$$

Using this knowledge, a healthy lung is modeled by generating a convex hull of the actual lung mask. Thickenings are normally limited to a small surface area, while other anatomical structures, e.g. ribs, have a more global effect on the shape of the lung. A convex hull of the whole lung mask not only removes thickenings, but yields an anatomically strongly altered lung model.

A concept called a sliding convex hull is introduced to model the healthy lung. Instead of computing the convex hull of the complete lung $\operatorname{conv}\left(\boldsymbol{R}_{L}\right)$, the healthy lung $\hat{\boldsymbol{R}}_{\boldsymbol{L}}$ is modeled, calculating the convex hull for each slice using $n$ surrounding slices in each direction. In a closed form, this can be expressed by

$$
\hat{\boldsymbol{R}}_{\boldsymbol{L}}=\bigcup_{\zeta=1}^{Z} \operatorname{conv}\left(\boldsymbol{R}_{\boldsymbol{L}, \boldsymbol{z}}\right) \cap\{i=(x, y, z): i \in \boldsymbol{R} ; z=\zeta\},
$$

where $\boldsymbol{R}_{\boldsymbol{L}, \boldsymbol{z}}=\left\{\boldsymbol{i}=(x, y, z): \boldsymbol{i} \in \boldsymbol{R}_{\boldsymbol{L}} ;|z-\zeta|<=n\right\}$ are the surrounding slices. Parameter $n$ controls the spatial extension of the considered thickenings. The use of $n=1$ limits the approach to a two-dimensional slice by slice analysis, and $n=Z$ is identical with computing a convex hull of the whole lung. The results for applying the sliding convex hull with different values for $n$ are shown in Figure 4. The lung mask image is provided by the mapping

$$
\hat{S}(\boldsymbol{i})= \begin{cases}1, & \text { if } \boldsymbol{i} \in \hat{\boldsymbol{R}}_{\boldsymbol{L}} \\ 0, & \text { else }\end{cases}
$$

The principle is visualized in Figure 3 with the used range of slices $(z-n) \ldots(z+n)$ shown in blue and the actual slice $z$ shown in red.
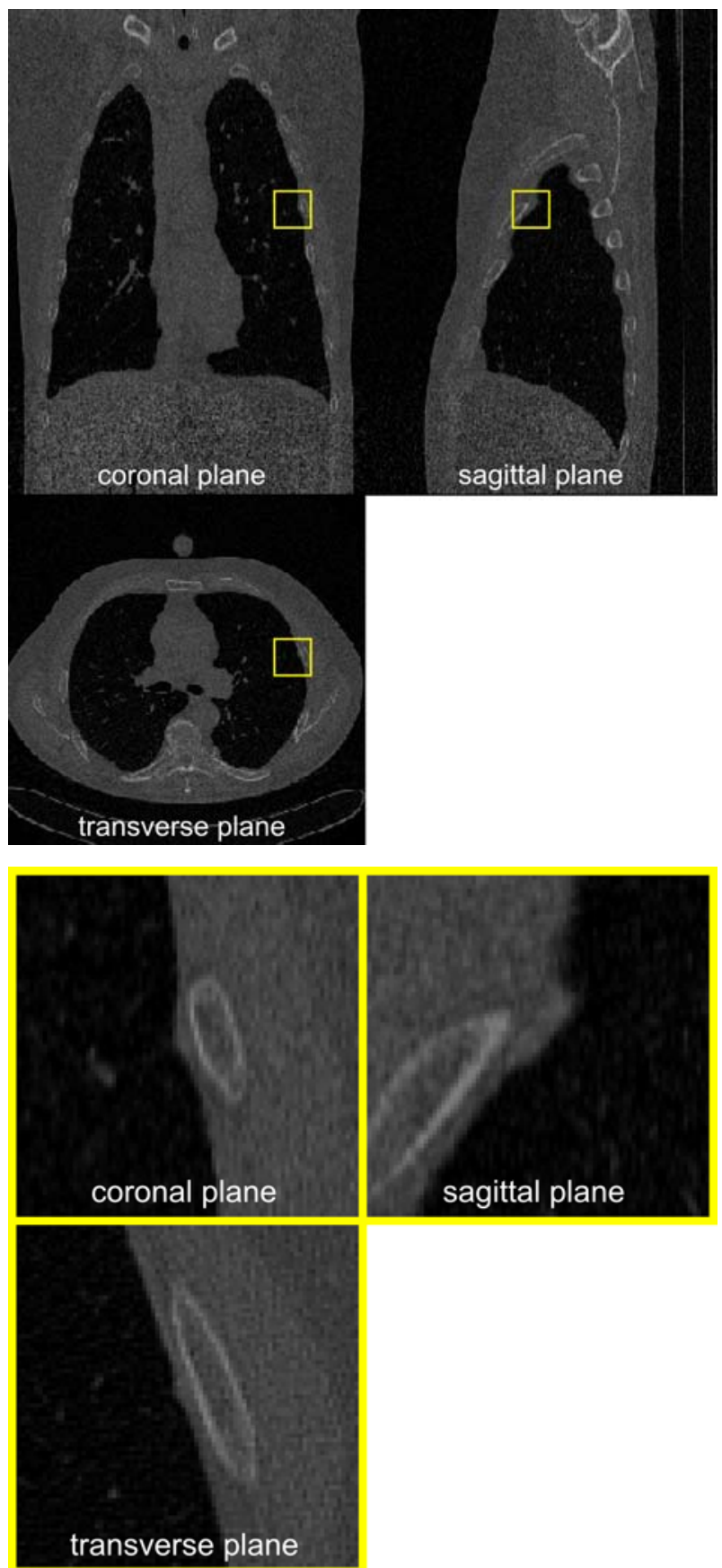

Fig. 2: Example of a pleural thickening not clearly visible in the transverse plane, but visible in the sagittal and coronal plane

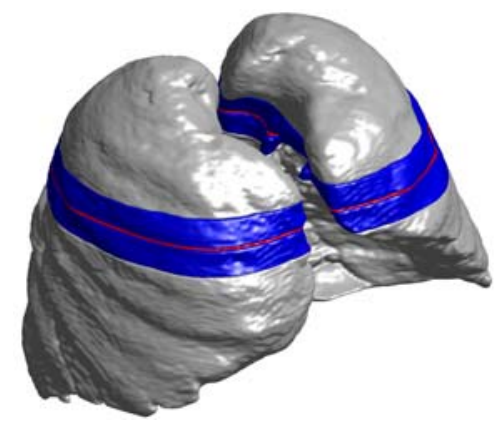

Fig. 3: The sliding convex hull principle 


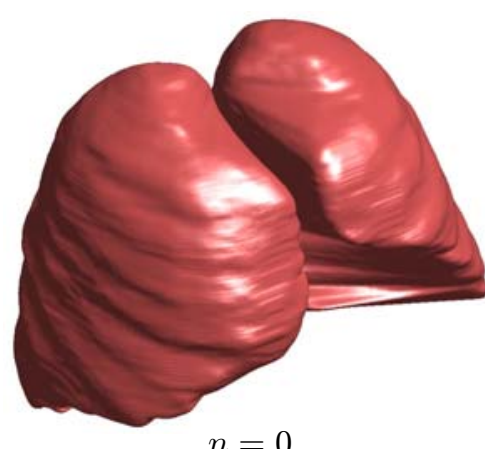

$n=0$
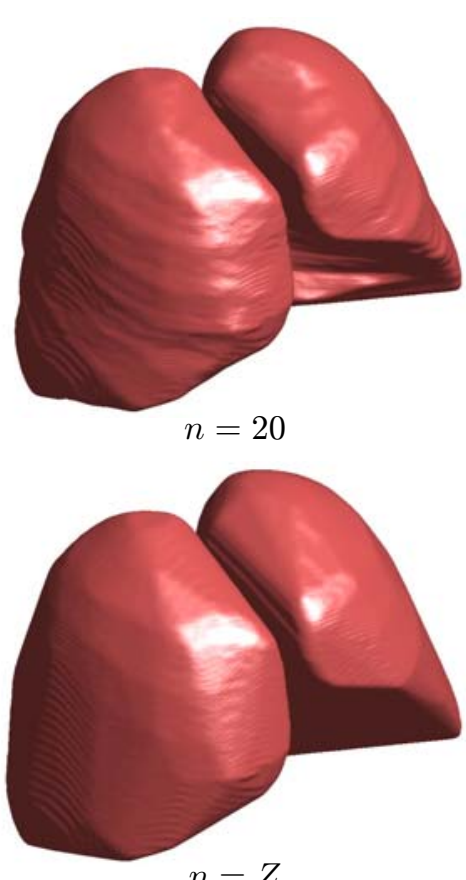

$n=Z$

Fig. 4: Results for different parameter settings of $n$

The differential image $\hat{S}(\boldsymbol{i}) \wedge \neg S(\boldsymbol{i}), \boldsymbol{i} \in \boldsymbol{R}$ includes all detectable thickenings. In addition, it contains other anatomical indentions as shown overlaid in Figure 5 , e.g. the ribs, the bronchial tree and the lobar fissure. These structures usually consist of huge segments. The removal of large segments would also discard all thickenings located in these anatomical regions. The thickenings however express their particular nature by almost never appearing on the bronchial tree and the lobar fissure [7]. All other regions of the lung surface contain only relatively flat anatomical structures like ribs, the backbone and lobar fissures. The undesired detection of these flat regions can be suppressed by virtually shrinking the healthy lung model, using the morphologic erosion operation $\ominus$. The shrunken model is given by

$$
\hat{S}_{e}=\hat{S} \ominus \boldsymbol{P}_{e}
$$

where $\boldsymbol{P}_{\boldsymbol{e}}$ is a sphere with the radius $(e+0.5)$ used as the structure element. The differences

$$
\Delta \boldsymbol{S}_{\boldsymbol{e}}(\boldsymbol{i})=\hat{S}_{e}(\boldsymbol{i}) \wedge \neg S(\boldsymbol{i}), \boldsymbol{i} \in \boldsymbol{R}
$$

using this model only contain structures indented more than $e$ voxels. Parameter $e$ is called the erosion level.

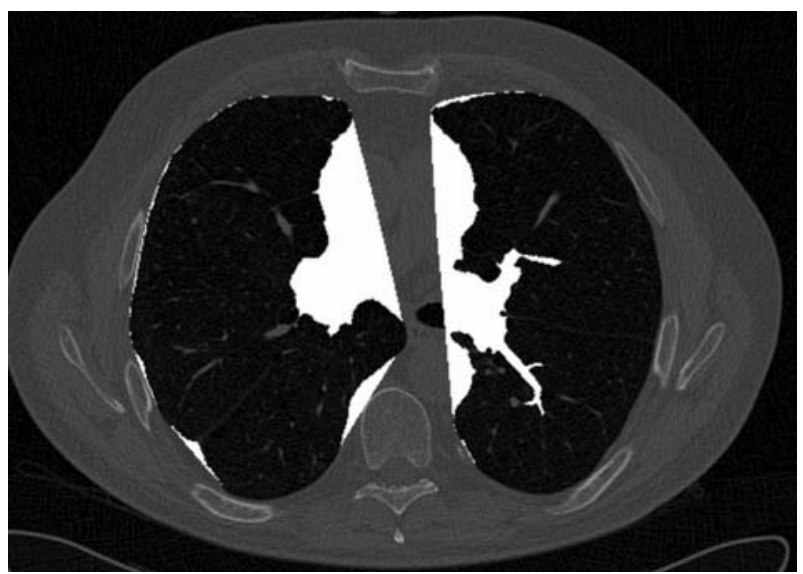

Fig. 5: An example of overlaid difference of modeled healthy lung mask and actual lung mask

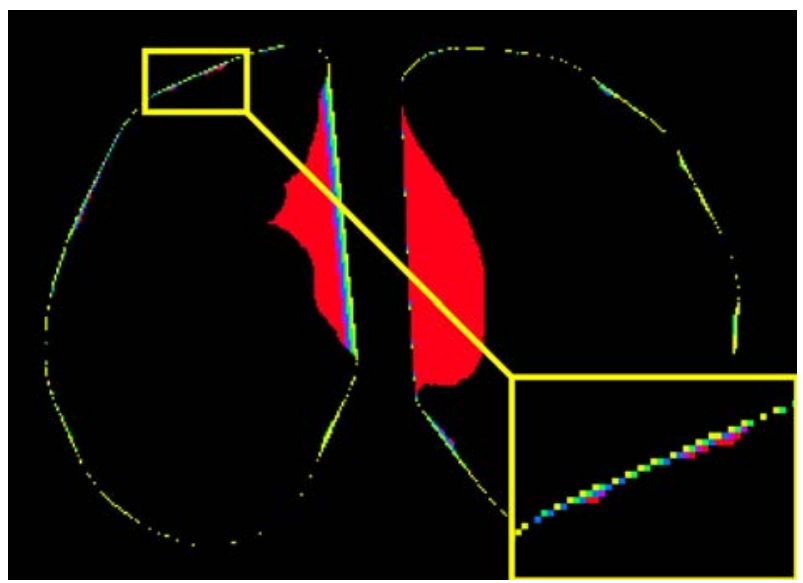

Fig. 6: Detected differences for different erosion levels in different colors

On each erosion level, flat anatomical structures with indention of $e$ or less are removed. Only large structures or thickenings with a higher indention remain. The large structures can be filtered out, as described below, and therefore only thickenings remain. In Figure 6 the differences between the eroded healthy lung model and the actual lung are visualized in different colors. Each voxel has a color depending on the highest erosion level, where it is contained in the differential image. The resulting segments have to be classified as thickenings or as normal anatomical structures. This is realized by filtering the segments depending on their size and depending on their CT number. Thickenings are usually from $5 \mathrm{~mm}^{3}$ to $2000 \mathrm{~mm}^{3}$ in volume, hence segments with more than $10000 \mathrm{~mm}^{3}$ can safely be classified as anatomical structures. While fat typically has a CT number in the range from $-220 \mathrm{HU}$ to $-50 \mathrm{HU}$, pleural mesothelioma is a tumorous connective tissue similar 
to muscle, whose Hounsfield Unit ranges between 20 and $60 \mathrm{HU}$. Therefore only segments including more than $10 \%$ of tissue with $10 \mathrm{HU}$ or higher are classified as thickenings. After filtering the differences $\Delta \boldsymbol{S}_{\boldsymbol{e}}(\boldsymbol{i})$, the final result of thickening detection is achieved by merging the detected thickenings from each erosion level, using

$$
\Delta \boldsymbol{S}(\boldsymbol{i})=\bigcup_{e=1}^{E} \text { filter }(\Delta \boldsymbol{S}(\boldsymbol{i}))
$$

where $E$ is the maximum expected indention depth of normal anatomical structures.

\section{Notes on implementation}

In this section we focus on the computationally intensive parts of the implementation. A complex part is the determination of the convex hull, which is performed for each slice. Only the surface of the lung mask contains points potentially contained in the subset defining the convex hull. Thus, a simple binary edge detector is applied to the lung mask $S$ to reduce the number of considered voxels that are used to calculate the convex hull. The calculation itself is performed using the Quickhull Algorithm for Convex Hulls [8]. Generating the healthy lung model requires fast voxelization of the triangles $\boldsymbol{G}=\left\{(i, j, k): i, j, k \in \hat{\boldsymbol{R}}_{\boldsymbol{L}}\right\}$, describing the convex hull. To reduce the considered number of triangles $\boldsymbol{G}{ }^{\prime}{ }_{\boldsymbol{\zeta}}$ in the slice $\zeta$, only triangles whose vertices $\boldsymbol{i}=\left(x_{i}, y_{i}, z_{i}\right), \boldsymbol{j}=\left(x_{j}, y_{j}, z_{j}\right), \boldsymbol{k}=\left(x_{k}, y_{k}, z_{k}\right)$ are all located in this slice $\left(z_{i}=z_{j}=z_{k}=\zeta\right)$ or intersect the slice $\left(\left(z_{i}<\zeta \vee z_{j}<\zeta \vee z_{k}<\zeta\right) \wedge\left(z_{i}>\right.\right.$ $\left.\left.\zeta \vee z_{j}>\zeta \vee z_{k}>\zeta\right)\right)$ are regarded. Voxelization is realized by recursively splitting the triangles, until their edge length is below 0.5 voxels. The resulting triangle edges cover all voxels of the lung surface. The resulting algorithm is highly efficient for parallel computing. Another computationally intensive task is the erosion of the lung model, which depends on the size of the structuring element. To reduce the slice of this structuring element, the erosion of level $e$ is approximated by erosion of level 1 applied to the results of the erosion from level $(e-1)$.

\section{Results}

The experiments on thickening detection are carried out using image data from four different patients. To evaluate the results, we measure both the totally detected volume of all thickenings, and the number of detected thickenings, for each patient. The results are plotted in Figure 7 for volume of the thickenings and in Figure 8 for the number of thickenings. Both metrics are measured depending on parameter $n$, which describes the size of the sliding convex hull.

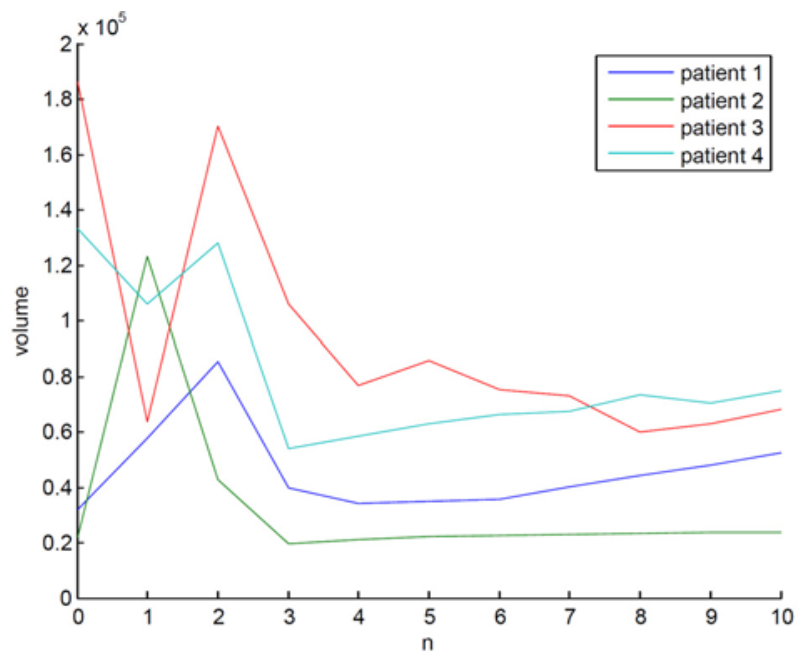

Fig. 7: Totally detected thickening volume depending on the size of the sliding convex hull

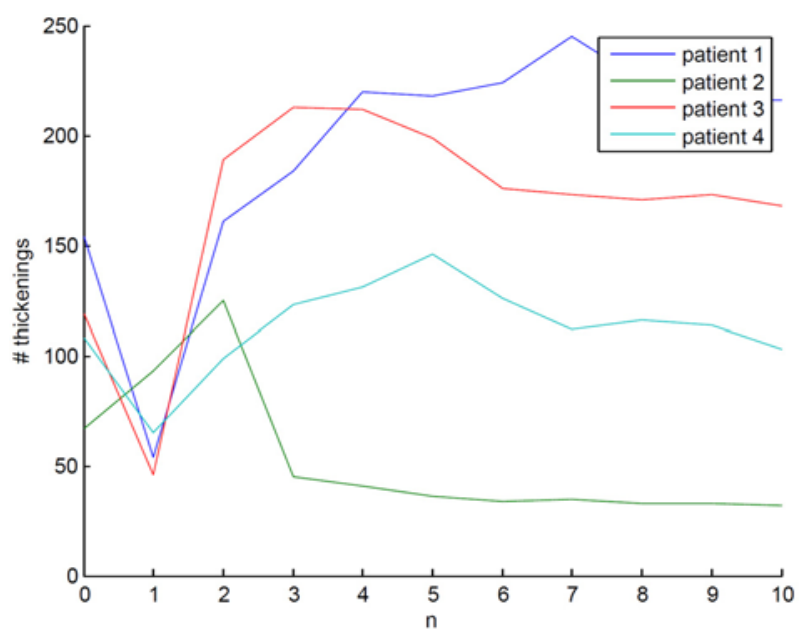

Fig. 8: Detected number of thickenings depending on the size of the sliding convex hull

\section{Discussion}

Neither an exact number of thickenings nor the thickening volume could be determined because of the inter- and intra-reader variances. Therefore only a interpretational evaluation is performed. Obviously the detected number of thickenings and the volume both fluctuate for $n<4$ and tend to a constant value for higher values of $n$. Unstable results occur in the range where the method has somehow a more $2 \mathrm{D}$ like behavior. This behavior results an inappropriate connectivity of the detected segments in the $z$ direction. Thus, on the one hand large anatomical structures could not be detected and be filtered out and, on the other hand, related segments could not be assigned to the same thickening. Another obvious effect is the increasing volume for increasing $n$, which can be explained by a growing convex hull. This is caused by the increasing effect of bulges outside the lung surface for increasing $n$. 


\section{Summary and future work}

We have presented a slice based approach for detecting pleural thickenings, including 3D information on the surrounding lung surface. The detected structures are automatically refined and filtered using stepwise erosion of a modelled healthy lung. Though this approach leads to an optically plausible result for the shape of the extracted thickenings, the method still focuses on detection. The exact boundaries of the thickenings need to be refined in further processing steps. For example the rear of the thickenings is currently interpolated only by applying a convex hull, which does not consider the surrounding lung curvature. Approaches like [9] promise more accurate interpolation of the rear of the thickenings.

\section{Acknowledgement}

The project is supported by German Social Accident Insurance (DGUV), project number FF-FB0148. We would like to thank Prof. Dr.-Ing. Til Aach, head of the Institute of Imaging and Computer Vision, RWTH Aachen University, Germany for supervising this project, and also Prof. Dr. med. Thomas Kraus, director of the Institute and Out-patient Clinic of Occupational Medicine, University Hospital Aachen, Germany for supervising and also for providing us with medical image data, background knowledge and evaluations.

\section{References}

[1] Pistolesi, M., Rusthoven, J.: Malignant Pleural Mesothelioma: Update, Current Management, and Newer Therapeutic Strategies, Chest, 2004, vol. 126, no. 4, pp. 1318-1329.

[2] Carl, T. M. I.: Interreadervarianz bei der HRCTund $C X R$-Befundung in einer Längsschnittstudie bei Ehemals Asbeststaubexponierten Personen. Ph.D. thesis, Medizinische Fakultät, RWTH Aachen, 2004.

[3] Chaisaowong, K., Knepper, A., Kraus, T., Aach, T.: Application of Supervised Rangeconstrained Thresholding to Extract Lung Pleura for Automated Detection of Pleural Thickenings from Thoracic CT Images. Proc. SPIE Medical Imaging: Computer-Aided Diagnosis, San Diego, CA, 2007, vol. 6 514, p. 65143M.

[4] Saekor, N., Roongruangsorakarn, S., Chaisaowong, K., Kraus, T., Aach, T.: 3D Modeling of Detected Pleural Thickenings Through Thin Plate Spline Interpolation. In Electrical Engineering/Electronics, Computer, Telecommunications and Information Technology, ECTI-CON, 2009, vol. 02, pp. 1106-1109.

[5] El-Baz, A., Gimel'Farb, G., Falk, R., ElGhar, M. A.: A Novel Approach for Automatic Follow-up of Detected Lung Nodules. ICIP, San Antonio, TX, 2007, vol. 5, pp. V-501-V-504.

[6] Chaisaowong, K., Bross, B., Knepper, A., Kraus, T., Aach, T.: Detection and Follow-up Assessment of Pleural Thickenings from 3D CT Data. ECTI-CON, 2008, vol. I, pp. 489-492.

[7] Chaisaowong, K., Jäger, P., Vogel, S., Knepper, A., Kraus, T., Aach, T.: Computer-assisted Diagnosis for Early Stage Pleural Mesothelioma: Towards Automated Detection and Quantitative Assessment of Pleural Thickenings from Thoracic CT Images. Methods of Information in Medicine, 2007, vol. 46(3), pp. 324-331.

[8] Bradford Barber, C., Dobkin, D. P., Huhdanpaa, H.: The Quickhull Algorithm for Convex Hulls. ACM Transactions on Mathematical Software, 1996, vol. 22, no. 4, pp. 469-483.

[9] Liepa, P.: Filling Holes in Meshes. Proceedings of the 2003 Eurographics/ACM SIGGRAPH symposium on Geometry processing, Aire-la-Ville, Switzerland, 2003, SGP '03, pp. 200-205.

\section{About the authors}

Peter Faltin was born in 1983 in Cologne, Germany. He studied Computer Engineering at RWTH Aachen University (Germany) and received his Dipl.Ing. in 2010. Currently, he is a PhD student at the Institute of Imaging \& Computer Vision at RWTH Aachen University, where he is working on the "Computer-Aided Early Diagnosis of Pleuramesothelioms" project. His research focuses on medical image processing, signal processing and computer vision.

Kraisorn Chaisaowong was born in Thailand. After his Diplom-Ingenieur studies (Electrical Engineering and Information Technology) in Biomedical Engineering at the Institute of Biomedical Engineering, Karlsruhe Institute of Technology (Germany), and a research stay at the Nora Eccles Harrison Cardiovascular Research and Training Institute, University of Utah (USA), he ha been employed as a university lecturer at the King Mongkut's University of Technology North Bangkok (Thailand). Currently, he is a PhD student at the Institute of Imaging \& Computer Vision, RWTH Aachen University, where he is responsible for the "Computer-Aided Early Diagnosis of Pleuramesothelioma" project. 
Peter Faltin

E-mail: peter.faltin@lfb.rwth-aachen.de

Institute of Imaging and Computer Vision

RWTH Aachen University, Germany
Kraisorn Chaisaowong

E-mail: kraisorn.chaisaowong@lfb.rwth-aachen.de http://www.lfb.rwth-aachen.de/

Institute of Imaging and Computer Vision RWTH Aachen University, Germany

King Mongkut's University of Technology

North Bangkok, Thailand 